\title{
Life table parameters as indicator of potato Varieties susceptibility to infestation with Phthorimaea operculella (Zeller)
}

\author{
Abdallah, Y.E.Y; M. S. Abdel-Wahed and GH. A. Youssef \\ Department of Plant Protection, Fac. of Agric., Ain Shams Univ., Cairo, Egypt
}

\begin{abstract}
Life table studies for Phthorimaea operculella (Zeller) were carried out on four potato varieties, namely Atlas, Spunta, Simone and Nicola to evaluate the preferred variety for feeding $P h$. operculella larvae. The calculated biological parameters viz. Net reproductive Rate $\left(\mathrm{R}_{0}\right)$, Generation time $\left(\mathrm{G}_{\mathrm{t}}\right)$, Intrinsic Rate of Increase $\left(r_{\mathrm{m}}\right)$, Finite Rate of Increase $(\lambda)$ and Population Doubling Time $\left(D_{t}\right)$ indicate that Atlas proved to be the quite favorable for achieving the highest developmental and multiplication rates of Ph. operculella, followed by Simone and Spunta, meanwhile Nicola was the least favorable data figure in this respect were obtained.

The natural mortality figures; Apparent Mortality (AM), Real Mortality (RM), Indispensible Mortality (IM), Mortality-Survivor Ratio (MSR) and log population and $k$ factor for larvae reared on Atlas variety were 41.86, 36, 22.32, 4.42, 2.54 and 0.24 respectively, the values $32.89,25,13.72,1.55,2.48$ and 0.17 were obtained for Simone, the values 43.75, 35, 21.77, 3.11, 2.50 and 0.25 for Spunta and 51.19, 43, 22.02 and 5.51for Nicola.
\end{abstract}

For pupae similar natural mortality trends were observed when $\mathrm{Ph}$. operculella larvae were fed on Atlas, 38, 19, 19, 3.77, 2.30 and 0.21, while these values were $45.10,23,23,2.6,2.31$ and 0.26 for Simone. The corresponding values on Spunta were $37.78,17,17,2.43,5$ and 2.21 and 48.78, 20, 20 and 5 for Nicola.

It could be concluded that Nicola is the least susceptible variety for infestation; while Atlas is the most favorable variety for feeding Ph. operculella for research purposes.

Keywords: Susceptibility, Varieties, Life tables, Potato tuber moth, Phthorimaea operculella.

\section{INTRODUCTION}

Potato tuber moth, Phthorimaea operculella (Zeller) is one of the most significant insect pests attacking potato tubers which are considered the most important food crop all- over the world. This pest causes reliable damage to potato plants in the field and great losses in quality and quantity of the yielded tubers during storage. The analysis of age-specific life tables is an important and is conducted through easy procedures based on biological parameters when the key factors governing the changes in the population dynamics. In the mean time, forecasting growth parameters; i.e. natality and mortality rates, provide a rational and predictive basis for pest control.

In this respect, many investigators studied the susceptibility of potato varieties to infestation with many insect pests including Ph. operculella either in the field or in the stores such as Fenemore (1980); Trivedi et al. (1994); Khattab et al. (1995); El-Saadany et al. (1998); Gurr \& Symington (1998); Ibrahim (2000); Chandel et al. (2001); Sileshi \& Terissa (2001); Ghazala (2005); Al-Taweel et al. (2006); Tiwari et al. (2006); Douches et al. (2010); Golizadeh \& Razmjou (2010); Horgan et al. (2010); Randon (2010) and Al-Omairy (2012). 
In general, the present study aimed to evaluate the susceptibility of certain potato varieties to infestation with $P h$. operculella; meanwhile determining the preferred potato variety for larval feeding.

\section{MATERIALS AND METHODS}

\section{Rearing techniques}

At harvest of potato yield of 2009 season, naturally infested tubers of four varieties namely Spunta, Nicola, Atlas and Simone were collected from the field for the establishment of a laboratory insect stock culture to provide target insect in all developmental stages required for laboratory studies. The infested potato tubers were medium sized and supplied with sound tubers and a $5 \mathrm{~cm}$ layer of wood sawdust to help successful pupation. These tubers were placed in nine wire gauze wooden rearing cages measuring $40 \times 40 \times 40 \mathrm{~cm}$. The culture was maintained at laboratory conditions of $27 \pm 2^{\circ} \mathrm{C}$ and $60 \pm 5 \%$ R.H. and a light regime of approximately $12 \mathrm{~h}$ daily light periodicity. The front of the rearing cage which served as a door for inserting selected tubers was provided with white cloth sleeves to facilitate daily examination; i.e. counting emerged moths and preventing their escape.

The newly emerged moths were collected by means of vacuum suction equipment, then sexed and grouped in 10 couples $(5 \hat{\jmath}+5$ + $)$ each in an oviposition cage consisting of a glass chimney measuring $17 \mathrm{~cm}$ in depth and $7-8.5 \mathrm{~cm}$ in diameter. The lower rim rested on the bottom of a Petri-dish lined with a disc of filter paper. The upper rim was covered with black muslin-upon which most of eggs were laid -fixed with a rubber band. Pieces of cotton stalks $6 \mathrm{~cm}$ in length soaked in $10 \%$ honey solution were supplied for moth feeding and were changed when needed. Deposited eggs were collected daily from the oviposition cages and kept in large test tubes covered with pieces of cotton. Newly hatched larvae were picked up by the aid of a moistened fine brush and transferred to potato tubers for feeding till pupation. Newly formed pupae were collected soon after pupation and placed in clean chimney glass cages. Daily examinations were carried out until adult emergence. Cages were cleaned and sterilized, saw dust was changed monthly.

\section{Life table studies}

A number of 400 newly deposited eggs were transferred from rearing cages using fine moist brush to ten plastic cups (400 eggs/variety of potato; i.e. 40 eggs/ replicate). Tested potato tubers of the four varieties with the same weight and size were offered to the larvae for feeding during the whole larval stage duration.

Newly formed pupae were transferred to a glass test tube covered with cotton till moth emerged. The newly emerged adults were sexed and transferred on the same day of emergence to an ovipositor glass chimney cage $(\hat{\sigma}++)$ in each cage which was covered with muslin and provided with piece of cotton soaked in $10 \%$ honey solution for feeding of moths.

The following biological aspects were measured; egg and larval duration and mortality, percentage of pupation, duration of pupal stage, percentage of adult emergence, adult longevity, female relative fecundity and specific fertility rates.

\section{Construction of life table data figures and estimating life table parameters}

Data obtained for evaluating the preferred potato variety for rearing $P h$. operculella survivors were used for constructing life tables according to Anderwartha and Birch (1984), Table (1).

The mortality data figures were estimated through the following:

* Apparent mortality $(\mathrm{AM} \%)=\left(\mathrm{dx}_{1} / \mathrm{lx}_{1}\right) \times 100,\left(\mathrm{dx}_{2} / \mathrm{lx}_{2}\right) \times 100 \ldots$ etc 
* Real mortality $(\mathrm{RM} \%)=\left(\mathrm{dx}_{1} / \mathrm{lx}_{1}\right) \times 100,\left(\mathrm{dx}_{2} / \mathrm{lx}_{2}\right) \times 100 \ldots$ etc

* Indispensable mortality (IM \%): this is that part of generation mortality that would not occur, should the mortality factor in question be removed from the life system.

* Mortality-survivor ratio (MSR\%): this measure represents the increase in population that would have occurred if the factor in question had been absent. If the final population is multiplied by this ratio then the resulting value represents in individuals, the indispensable mortality due to that factor.

* Log population: the natural logarithm of the population.

$* k$ - value $=\log \mathrm{N}_{\mathrm{t}}-\log \mathrm{N}_{\mathrm{t}+1}$

Table 1: Age specific life table data figures expressed as definitions and corresponded formulae

\begin{tabular}{|c|c|c|}
\hline Symbol & Definitions & Formula \\
\hline $\mathbf{x}$ & Age ( in days ) & --- \\
\hline $\mathbf{I}_{\mathbf{x}}$ & Probability of an individual surviving to age $\mathrm{x}$. & \\
\hline $\mathbf{m}_{\mathbf{x}}$ & Reproductive expectation of a female at age $\mathrm{x}$. & 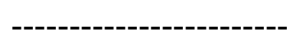 \\
\hline $\mathbf{R}_{\mathbf{0}}$ & $\begin{array}{l}\text { Net reproductive rate, number of daughters that replace an average female in } \\
\text { course of a generation. }\end{array}$ & $\mathbf{m}_{\mathbf{x}}$ \\
\hline $\mathbf{G}_{\mathbf{t}}$ & Mean generation time, mean of the period over which progeny are produced. & $\mathbf{G}_{\mathrm{t}}=\left(\sum \mathbf{x} \mathbf{l}_{\mathbf{x}} \mathbf{M}_{\mathbf{x}}\right) / \mathbf{R}_{\mathbf{0}}$ \\
\hline$r_{\mathrm{m}}$ & Intrinsic rate of increase, number of progeny produced per unit time & $r_{m}=\left(\log \mathrm{e}^{\mathrm{R} 0}\right) / \mathbf{G}_{\mathrm{t}}$ \\
\hline$\lambda$ & $\begin{array}{l}\text { Finite rate of increase, number of times a population double itself in unit } \\
\text { time. }\end{array}$ & $8 \mathrm{rn}$ \\
\hline $\mathbf{D}_{\mathrm{t}}$ & Generation doubling time. & $D_{t}=\left(\log e^{2}\right) / r_{m}$ \\
\hline
\end{tabular}

\section{RESULTS AND DISCUTION}

The following parameters were considered.

\section{a- Survival and fecundity rates}

Data presented in Tables (2-5) and Fig. (1) summarize the calculated life table parameters of potato tuber moth when larvae were fed on tubers of Atlas, Spunta, Simone and Nicola varieties, respectively. However data indicate reliable differences in the biological parameters between varieties' means.
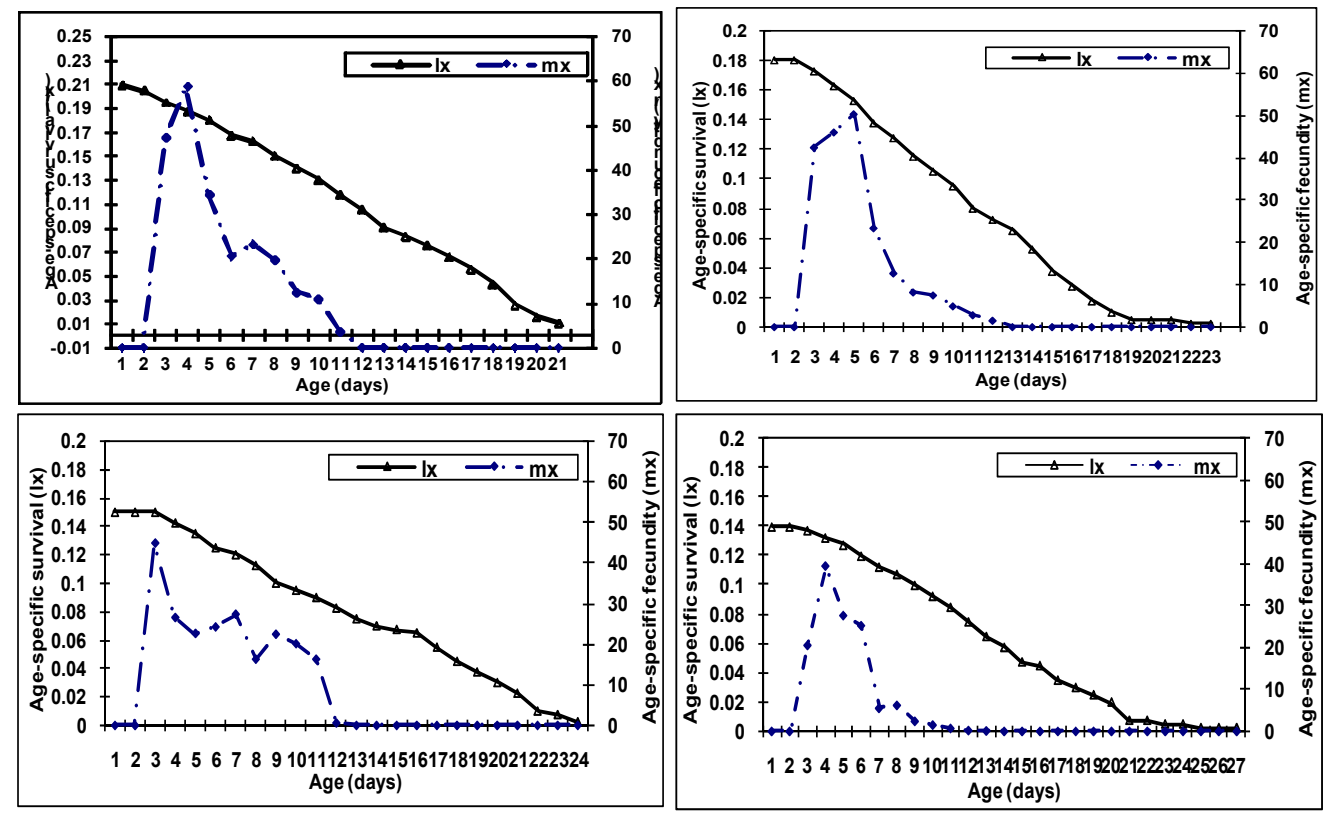

Fig.1: Age-specific survivorship $\left(1_{\mathrm{x}}\right)$ and fecundity $\left(\mathrm{m}_{\mathrm{x}}\right)$ of Ph. operculella on four varieties of potatoes. 
Data in Table (2) reveal that female moths emerging from larvae fed on Atlas tubers demonstrated the shortest longevity duration (21 days) and the highest agespecific fecundity rate of 229.8 eggs/female; meanwhile durations of immature stages were completed in 21 days. Females started oviposition after 2 days from emergence and lasted for 9 days while the post-oviposition period lasted for about 10 days. Net reproductive rate $\left(R_{0}\right)$ was 40.01 eggs/female. Meanwhile duration of generation $\left(G_{t}\right)$ was 26.17 days after which death occurred. The total life span lasted for 42 days (Table 6).

Nicola variety, however, demonstrated the longest life span duration thus completing one generation in 52 days. The immature stages lasted for 25 days. Female longevity lasted 27 days. Fecundity rate was 129.3 eggs/female. Net reproductive rate was 16.33 eggs/day. Generation time was 29.85 days (Tables $5 \& 6$ ).

Potato tuber moth larvae when fed on Simone and Spunta demonstrated a moderate duration data figures thus completing one generation within 43 and 47 days, respectively. Meanwhile, the immature stages lasted for $19 \& 24$ days on both tested verities. Female longevity lasted $23 \& 22$ days, respectively. Numbers of $220.1 \& 200$ eggs/female; 27.23 and 29.87 eggs/female were recorded for female fecundity and net reproductive rate, respectively; while mean duration of the generation was $24.93 \&$ 28.82 days, respectively (Tables $3,4 \& 6$ ).

Table 2: Life table parameters expressed as number of survivors $\left(l_{\mathrm{x}}\right)$ and fecundity rates $\left(\mathrm{m}_{\mathrm{x}}\right)$ of $P h$. operculella when larvae were fed on potato tubers variety Atlas for one generation

\begin{tabular}{|c|c|c|c|c|c|c|}
\hline Stage & $\begin{array}{c}\text { Age } \\
\text { (days) } \\
\mathbf{x} \\
\end{array}$ & $\begin{array}{c}\text { No. of } \\
\text { observation }\end{array}$ & $\begin{array}{c}\text { Survivorship } \\
\mathbf{l}_{\mathbf{x}}\end{array}$ & $\begin{array}{l}\text { Fecundity } \\
\mathbf{m}_{\mathbf{x}}\end{array}$ & $\mathbf{l}_{\mathbf{x}} \mathbf{m}_{\mathbf{x}}$ & $\mathbf{l}_{\mathbf{x}} \mathbf{m}_{\mathbf{x}} \mathbf{x}$ \\
\hline Eggs & 3 & 400 & 1.0000 & & & \\
\hline Larvae & 12 & 344 & 0.8600 & & & \\
\hline Pupae & 6 & 200 & 0.5000 & & & \\
\hline \multirow[t]{23}{*}{ Female } & 22 & 84 & 0.2100 & 0.0 & 0.00 & 0.00 \\
\hline & 23 & 82 & 0.2050 & 0.0 & 0.00 & 0.00 \\
\hline & 24 & 78 & 0.1950 & 47.2 & 9.20 & 220.89 \\
\hline & 25 & 75 & 0.1875 & 58.6 & 10.99 & 274.69 \\
\hline & 26 & 72 & 0.1800 & 34.2 & 6.16 & 160.06 \\
\hline & 27 & 67 & 0.1675 & 20.5 & 3.43 & 92.71 \\
\hline & 28 & 65 & 0.1625 & 23.1 & 3.75 & 105.11 \\
\hline & 29 & 60 & 0.1500 & 19.6 & 2.94 & 85.26 \\
\hline & 30 & 56 & 0.1400 & 12.4 & 1.74 & 52.08 \\
\hline & 31 & 52 & 0.1300 & 10.7 & 1.39 & 43.12 \\
\hline & 32 & 47 & 0.1175 & 3.5 & 0.41 & 13.16 \\
\hline & 33 & 42 & 0.1050 & 0.0 & 0.00 & 0.00 \\
\hline & 34 & 36 & 0.0900 & 0.0 & 0.00 & 0.00 \\
\hline & 35 & 33 & 0.0825 & 0.0 & 0.00 & 0.00 \\
\hline & 36 & 30 & 0.0750 & 0.0 & 0.00 & 0.00 \\
\hline & 37 & 26 & 0.0650 & 0.0 & 0.00 & 0.00 \\
\hline & 38 & 22 & 0.0550 & 0.0 & 0.00 & 0.00 \\
\hline & 39 & 17 & 0.0425 & 0.0 & 0.00 & 0.00 \\
\hline & 40 & 10 & 0.0250 & 0.0 & 0.00 & 0.00 \\
\hline & 41 & 6 & 0.0150 & 0.0 & 0.00 & 0.00 \\
\hline & 42 & 4 & 0.0100 & 0.0 & 0.00 & 0.00 \\
\hline & Total & & & 229.8 & $\mathrm{R}_{0}=$ & 1047.08 \\
\hline & Mean & & & 10.94 & 40.01 & \\
\hline
\end{tabular}

$\mathrm{G}_{\mathrm{t}}=1047.08 / 40.01=26.17$ 
Table 3: Life table parameters expressed as number of survivors $\left(l_{\mathrm{x}}\right)$ fecundity rates $\left(\mathrm{m}_{\mathrm{x}}\right)$ of $P h$. operculella when larvae were fed on potato tubers variety Simone for one generation.

\begin{tabular}{|c|c|c|c|c|c|c|}
\hline Stage & $\begin{array}{c}\text { Age } \\
\text { (days) } \\
\text { X }\end{array}$ & $\begin{array}{c}\text { No. of } \\
\text { observation }\end{array}$ & $\begin{array}{c}\text { Survivorship } \\
l_{x}\end{array}$ & $\begin{array}{c}\text { Fecundity } \\
\mathbf{m}_{\mathbf{x}}\end{array}$ & $\mathbf{l}_{\mathrm{x}} \mathbf{m}_{\mathbf{x}}$ & $\mathbf{l}_{\mathbf{x}} \mathbf{m}_{\mathbf{x}} \mathbf{x}$ \\
\hline Egg & 3 & 400 & 1.0000 & & & \\
\hline Larvae & 11 & 304 & 0.7600 & & & \\
\hline Pupae & 5 & 204 & 0.5100 & & & \\
\hline \multirow[t]{26}{*}{ Female } & 20 & 60 & 0.1500 & 0.0 & 0.00 & 0.00 \\
\hline & 21 & 60 & 0.1500 & 0.0 & 0.00 & 0.00 \\
\hline & 22 & 60 & 0.1500 & 44.6 & 6.69 & 147.18 \\
\hline & 23 & 57 & 0.1425 & 26.4 & 3.76 & 86.53 \\
\hline & 24 & 54 & 0.1350 & 22.5 & 3.04 & 72.90 \\
\hline & 25 & 50 & 0.1250 & 24.1 & 3.01 & 75.31 \\
\hline & 26 & 48 & 0.1200 & 27.2 & 3.26 & 84.86 \\
\hline & 27 & 45 & 0.1125 & 16.2 & 1.82 & 49.21 \\
\hline & 28 & 40 & 0.1000 & 22.3 & 2.23 & 62.44 \\
\hline & 29 & 38 & 0.0950 & 20 & 1.90 & 55.10 \\
\hline & 30 & 36 & 0.0900 & 16.2 & 1.46 & 43.74 \\
\hline & 31 & 33 & 0.0825 & 0.6 & 0.05 & 1.53 \\
\hline & 32 & 30 & 0.0750 & 0.0 & 0.00 & 0.00 \\
\hline & 33 & 28 & 0.0700 & 0.0 & 0.00 & 0.00 \\
\hline & 34 & 27 & 0.0675 & 0.0 & 0.00 & 0.00 \\
\hline & 35 & 26 & 0.0650 & 0.0 & 0.00 & 0.00 \\
\hline & 36 & 22 & 0.0550 & 0.0 & 0.00 & 0.00 \\
\hline & 37 & 18 & 0.0450 & 0.0 & 0.00 & 0.00 \\
\hline & 38 & 15 & 0.0375 & 0.0 & 0.00 & 0.00 \\
\hline & 39 & 12 & 0.0300 & 0.0 & 0.00 & 0.00 \\
\hline & 40 & 9 & 0.0225 & 0.0 & 0.00 & 0.00 \\
\hline & 41 & 4 & 0.0100 & 0.0 & 0.00 & 0.00 \\
\hline & 42 & 3 & 0.0075 & 0.0 & 0.00 & 0.00 \\
\hline & 43 & 1 & 0.0025 & 0.0 & 0.00 & 0.00 \\
\hline & Total & & & 220.1 & $\mathrm{R}_{0}=$ & 67880 \\
\hline & Mean & & & 9.17 & 27.23 & $0 / 8.80$ \\
\hline
\end{tabular}

$\mathrm{G}_{\mathrm{t}}=\overline{678.80 / 27.23=24.93}$

Table 4: Life table parameters expressed as number of survivors $\left(l_{\mathrm{x}}\right)$ and fecundity rates $\left(\mathrm{m}_{\mathrm{x}}\right)$ of $P h$. operculella when larvae were fed on potato tubers variety Spunta for one generation

\begin{tabular}{|c|c|c|c|c|c|c|}
\hline Stage & $\begin{array}{c}\text { Age } \\
\text { (days) } \\
\text { X }\end{array}$ & $\begin{array}{c}\text { No. of } \\
\text { observation }\end{array}$ & $\begin{array}{c}\text { Survivorship } \\
\mathbf{l}_{\mathrm{x}}\end{array}$ & $\begin{array}{c}\text { Fecundity } \\
\mathbf{m}_{\mathbf{x}}\end{array}$ & $\mathbf{l}_{\mathbf{x}} \mathbf{m}_{\mathbf{x}}$ & $\mathbf{l}_{\mathbf{x}} \mathbf{m}_{\mathbf{x}} \mathbf{x}$ \\
\hline Egg & 3 & 400 & 1.0000 & & & \\
\hline Larvae & 14 & 320 & 0.8000 & & & \\
\hline Pupae & 7 & 180 & 0.4500 & & & \\
\hline \multirow[t]{25}{*}{ Female } & 25 & 72 & 0.1800 & 0.0 & 0.00 & 0.00 \\
\hline & 26 & 72 & 0.1800 & 0.0 & 0.00 & 0.00 \\
\hline & 27 & 69 & 0.1725 & 42.5 & 7.33 & 197.94 \\
\hline & 28 & 65 & 0.1625 & 46.1 & 7.49 & 209.75 \\
\hline & 29 & 61 & 0.1525 & 50.4 & 7.69 & 222.89 \\
\hline & 30 & 55 & 0.1375 & 23.4 & 3.22 & 96.53 \\
\hline & 31 & 51 & 0.1275 & 12.7 & 1.62 & 50.20 \\
\hline & 32 & 46 & 0.1150 & 8.2 & 0.94 & 30.18 \\
\hline & 33 & 42 & 0.1050 & 7.5 & 0.79 & 25.99 \\
\hline & 34 & 38 & 0.0950 & 4.9 & 0.46 & 15.83 \\
\hline & 35 & 32 & 0.0800 & 2.8 & 0.22 & 7.84 \\
\hline & 36 & 29 & 0.0725 & 1.5 & 0.12 & 3.92 \\
\hline & 37 & 26 & 0.0650 & 0.0 & 0.00 & 0.00 \\
\hline & 38 & 21 & 0.0525 & 0.0 & 0.00 & 0.00 \\
\hline & 39 & 15 & 0.0375 & 0.0 & 0.00 & 0.00 \\
\hline & 40 & 11 & 0.0275 & 0.0 & 0.00 & 0.00 \\
\hline & 41 & 7 & 0.0175 & 0.0 & 0.00 & 0.00 \\
\hline & 42 & 4 & 0.0100 & 0.0 & 0.00 & 0.00 \\
\hline & 43 & 2 & 0.0050 & 0.0 & 0.00 & 0.00 \\
\hline & 44 & 2 & 0.0050 & 0.0 & 0.00 & 0.00 \\
\hline & 45 & 2 & 0.0050 & 0.0 & 0.00 & 0.00 \\
\hline & 46 & 1 & 0.0025 & 0.0 & 0.00 & 0.00 \\
\hline & 47 & 1 & 0.0025 & 0.0 & 0.00 & 0.00 \\
\hline & Total & & & 200 & $\mathrm{R}_{0}=$ & 861.06 \\
\hline & Mean & & & 8.69 & 29.87 & \\
\hline
\end{tabular}

$\mathrm{G}_{\mathrm{t}}=861.06 / 29.87=28.82$

\section{b- Intrinsic rate of increase $\left(r_{m}\right)$}

The population intrinsic rates $\left(r_{\mathrm{m}}\right)$ decreased from $0.474,0.450$ to 0.237 individuals/ female/day when larvae were fed on Simone, Spunta and Nicola. Meanwhile the highest value of intrinsic rate of increase 0.664 individuals/female/day was obtained when the larvae were reared on Atlas. The fore-mentioned results 
confirm that Atlas was the most suitable host variety as verified by $\mathrm{R}_{0}, \mathrm{G}_{\mathrm{t}}$ and $r_{\mathrm{m}}$ values (Table 6).

Table 5: Life table parameters expressed as number of survivors $\left(l_{\mathrm{x}}\right)$ fecundity rates $\left(\mathrm{m}_{\mathrm{x}}\right)$ of $P h$. operculella when larvae were fed on potato tubers variety Nicola for the fall generation

\begin{tabular}{|c|c|c|c|c|c|c|}
\hline Stage & $\begin{array}{c}\text { Age } \\
\text { (days) } \\
\text { X }\end{array}$ & $\begin{array}{c}\begin{array}{c}\text { No. of } \\
\text { observation }\end{array} \\
\end{array}$ & $\begin{array}{c}\text { Survivorship } \\
\mathbf{l}_{\mathrm{x}}\end{array}$ & $\begin{array}{c}\text { Fecundity } \\
\mathbf{m}_{\mathrm{x}}\end{array}$ & $\mathbf{l}_{\mathrm{x}} \mathbf{m}_{\mathrm{x}}$ & $\mathbf{l}_{\mathbf{x}} \mathbf{m}_{\mathbf{x}} \mathbf{x}$ \\
\hline Egg & 3 & 400 & 1.0000 & & & \\
\hline Larvae & 15 & 336 & 0.8400 & & & \\
\hline Pupae & 7 & 164 & 0.4100 & & & \\
\hline \multirow{29}{*}{ Female } & 26 & 56 & 0.1400 & 0.0 & 0.00 & 0.00 \\
\hline & 27 & 56 & 0.1400 & 0.0 & 0.00 & 0.00 \\
\hline & 28 & 55 & 0.1375 & 20.5 & 2.82 & 78.93 \\
\hline & 29 & 53 & 0.1325 & 39.4 & 5.22 & 151.39 \\
\hline & 30 & 51 & 0.1275 & 27.6 & 3.52 & 105.57 \\
\hline & 31 & 48 & 0.1200 & 25.2 & 3.02 & 93.74 \\
\hline & 32 & 45 & 0.1125 & 5.5 & 0.62 & 19.80 \\
\hline & 33 & 43 & 0.1075 & 6.2 & 0.67 & 21.99 \\
\hline & 34 & 40 & 0.1000 & 2.4 & 0.24 & 8.16 \\
\hline & 35 & 37 & 0.0925 & 1.5 & 0.14 & 4.86 \\
\hline & 36 & 34 & 0.0850 & 0.7 & 0.06 & 2.14 \\
\hline & 37 & 30 & 0.0750 & 0.2 & 0.01 & 0.56 \\
\hline & 38 & 26 & 0.0650 & 0.1 & 0.01 & 0.25 \\
\hline & 39 & 23 & 0.0575 & 0.0 & 0.00 & 0.00 \\
\hline & 40 & 19 & 0.0475 & 0.0 & 0.00 & 0.00 \\
\hline & 41 & 18 & 0.0450 & 0.0 & 0.00 & 0.00 \\
\hline & 42 & 14 & 0.0350 & 0.0 & 0.00 & 0.00 \\
\hline & 43 & 12 & 0.0300 & 0.0 & 0.00 & 0.00 \\
\hline & 44 & 10 & 0.0250 & 0.0 & 0.00 & 0.00 \\
\hline & 45 & 8 & 0.0200 & 0.0 & 0.00 & 0.00 \\
\hline & 46 & 3 & 0.0075 & 0.0 & 0.00 & 0.00 \\
\hline & 47 & 3 & 0.0075 & 0.0 & 0.00 & 0.00 \\
\hline & 48 & 2 & 0.0050 & 0.0 & 0.00 & 0.00 \\
\hline & 49 & 2 & 0.0050 & 0.0 & 0.00 & 0.00 \\
\hline & 50 & 1 & 0.0025 & 0.0 & 0.00 & 0.00 \\
\hline & 51 & 1 & 0.0025 & 0.0 & 0.00 & 0.00 \\
\hline & 52 & 1 & 0.0025 & 0.0 & 0.00 & 0.00 \\
\hline & Total & & & 129.3 & $\mathrm{R}_{0}=$ & 48730 \\
\hline & Mean & & & 4.79 & 16.33 & $40 \%$ \\
\hline
\end{tabular}

\section{c- Finite rate of increase $(\lambda)$}

When the finite rate of increase $(\lambda)$ values were worked out, it yielded 1.942, 1.606, 1.568 and 1.267 individuals/female/day when larvae were fed on Atlas, Simone, Spunta and Nicola, respectively. It is obvious from the obtained data that the highest finite rate of increase $(\lambda)$ of potato tuber moth was obtained for Atlas being 1.942 individuals/female/day and accordingly considered as the most preferred variety. The lowest preferred variety in this respect was Nicola (Table 6).

Table 6: Life table parameters of Ph. operculella larvae reared on four potato varieties

\begin{tabular}{cccccccc}
\hline Variety & $\begin{array}{c}\mathbf{x} \\
\text { days }\end{array}$ & $\begin{array}{c}\mathbf{m}_{\mathbf{x}} \\
\text { egg/day }\end{array}$ & $\begin{array}{c}\mathbf{R}_{\mathbf{0}} \\
\text { egg/female }\end{array}$ & $\begin{array}{c}\mathbf{G}_{\mathbf{t}} \\
\text { days }\end{array}$ & $\begin{array}{c}\boldsymbol{r}_{\mathrm{m}} \\
\text { individual/female }\end{array}$ & $\begin{array}{c}\boldsymbol{\lambda} \\
\text { individuals/female }\end{array}$ & $\begin{array}{c}\mathbf{D}_{\mathbf{t}} \\
\text { days }\end{array}$ \\
\hline Atlas & 42 & 10.94 & 40.01 & 26.17 & 0.664 & 1.942 & 1.308 \\
Spunta & 47 & 8.69 & 29.87 & 28.82 & 0.450 & 1.568 & 1.930 \\
Simone & 43 & 9.17 & 27.23 & 24.93 & 0.474 & 1.606 & 1.832 \\
Nicola & 52 & 4.79 & 16.33 & 29.85 & 0.237 & 1.267 & 3.665 \\
\hline
\end{tabular}

\section{d- Population doubling time $\left(D_{t}\right)$}

The population of potato tuber moth doubled once every $1.31,1.83,1.93 \& 3.66$ days when larvae were feed on Atlas, Simone, Spunta and Nicola tubers, respectively. 
It appears from the data in Table (6) that Atlas is the most preferred variety for potato tuber moth rearing because its population was doubled in the shortest time.

In general, the calculated biological parameters viz. $\mathrm{R}_{0}, \mathrm{G}_{\mathrm{t}}, r_{\mathrm{m}}, \lambda \& \mathrm{D}_{\mathrm{t}}$ indicate that Atlas proved to be the most favorable variety for achieving the highest developmental and reproduction rates for Ph. operculella, followed by Simone and Spunta. Meanwhile Nicola was the least favorable potato variety which is considered as the most tolerant variety for infestation with Ph. operculella. Abdel-Wahab et al. (1987) mentioned that generation time of Ph. operculella was $27.94 \& 78.7$ days during summer and winter, respectively. El-Saadany et al. (1998) also found that the generation time for this pest when reared at $30^{\circ} \mathrm{C}$ was 22.34 days.

\section{e- Natural mortality analysis}

When the values of different types of natural mortality namely, apparent mortality (AM), real mortality (RM), indispensable mortality (IM), mortality-survivor ratio (MSR), log population and $k$ value were estimated. Atlas variety proved to be the most preferred variety based on number of deposited eggs in one generation revealing its strong suitability for feeding. Mortality parameters are shown in Table (7). Similar trends were observed when the natural mortality in larvae and pupae expressed by mortality index. Hence Atlas variety again proved to be highly preferred for feeding and accordingly harbored the lowest percentages of natural mortality figures (Table, 7). The respective values on Simone, Spunta and Nicola varieties are given in Tables (8-10).

For Ph. operculella pupae natural mortality trend AM, RM, IM, Log pop and $K$ when fed as larvae on the four varieties are also are also given in Tables (7-10).

Table 7: Changes in natural mortality of the developmental stages of potato tuber moth when larvae were fed on tubers of Atlas variety

\begin{tabular}{|c|c|c|c|c|c|c|c|}
\hline \multicolumn{8}{|c|}{ Developmental stage } \\
\hline Age class & Egg & & Larvae & & Pupae & & Adults \\
\hline $\mathbf{x}$ & $(0-3)$ & & $(4-15)$ & & $(16-21)$ & & $(22-42)$ \\
\hline $\mathbf{l}_{\mathrm{x}}$ & 400 & & 344 & & 200 & & 124 \\
\hline $\mathbf{d}_{\mathbf{x}}$ & & 56 & & 144 & & 76 & \\
\hline A.M. \% & 14 & & 41.86 & & 38 & & \\
\hline R.M.\% & 14 & & 36.00 & & 19 & & \\
\hline I.M.\% & 5.04 & & 22.32 & & 19 & & \\
\hline M.S.R.\% & 1 & & 4.42 & & 3.77 & & \\
\hline Log population & 2.60 & & 2.54 & & 2.30 & & 2.09 \\
\hline$k$ - value & & 0.06 & & 0.24 & & 0.21 & \\
\hline
\end{tabular}

Numbers between parentheses represent the range of duration in days

Table 8: Changes in natural mortality of the developmental stages of potato tuber moth when larvae were fed on tubers of Simone variety

\begin{tabular}{|c|c|c|c|c|c|c|c|}
\hline \multicolumn{8}{|c|}{ Developmental stage } \\
\hline Age class & Egg & & Larvae & & Pupae & & Adults \\
\hline $\mathbf{x}$ & $(0-3)$ & & $(4-14)$ & & $(15-19)$ & & $(20-43)$ \\
\hline $\mathbf{l}_{\mathrm{x}}$ & 400 & & 304 & & 204 & & 112 \\
\hline $\mathbf{d}_{\mathrm{x}}$ & & 96 & & 100 & & 92 & \\
\hline A.M.\% & 24 & & 32.89 & & 45.10 & & \\
\hline R.M.\% & 24 & & 25 & & 23 & & \\
\hline I.M.\% & 8.84 & & 13.72 & & 23 & & \\
\hline M.S.R.\% & 1 & & 1.55 & & 2.6 & & \\
\hline Log population & 2.60 & & 2.48 & & 2.31 & & 2.05 \\
\hline$k$-value & & 0.12 & & 0.17 & & 0.26 & \\
\hline
\end{tabular}

Numbers between parentheses represent the range of duration in days 
Table 9: Changes in natural mortality of the developmental stages of potato tuber moth when larvae were fed on tubers of Spunta variety

\begin{tabular}{|c|c|c|c|c|c|c|}
\hline \multicolumn{7}{|c|}{ Developmental stage } \\
\hline Age class & Egg & Larvae & & Pupae & & Adults \\
\hline $\mathbf{x}$ & $(0-3)$ & $(4-17)$ & & $(18-24)$ & & $(25-47)$ \\
\hline $\mathbf{l}_{\mathbf{x}}$ & 400 & 320 & & 180 & & 112 \\
\hline$d_{x}$ & & 80 & 140 & & 68 & \\
\hline A.M.\% & 20 & 43.75 & & 37.78 & & \\
\hline R.M.\% & 20 & 35 & & 17 & & \\
\hline I.M.\% & 7 & 21.77 & & 17 & & \\
\hline M.S.R.\% & 1 & 3.11 & & 2.43 & & \\
\hline Log population & 2.60 & 2.50 & & 2.25 & & 2.05 \\
\hline$k$-value & & 0.1 & 0.25 & & 0.2 & \\
\hline
\end{tabular}

Numbers between parentheses represent the range of duration in days

Table 10: Changes in natural mortality of the developmental stages of potato tuber moth when larvae were fed on tubers of Nicola variety

\begin{tabular}{|c|c|c|c|c|c|c|c|}
\hline \multicolumn{8}{|c|}{ Developmental stage } \\
\hline Age class & Egg & & Larvae & & Pupae & & Adults \\
\hline $\mathbf{x}$ & $(0-3)$ & & $(4-18)$ & & $(19-25)$ & & $(26-52)$ \\
\hline $\mathbf{l}_{\mathbf{x}}$ & 400 & & 336 & & 164 & & 84 \\
\hline $\mathbf{d}_{\mathbf{x}}$ & & 64 & & 172 & & 80 & \\
\hline А.М.\% & 16 & & 51.19 & & 48.78 & & \\
\hline R.M.\% & 16 & & 43 & & 20 & & \\
\hline I.M.\% & 4 & & 22.02 & & 20 & & \\
\hline M.S.R.\% & 1 & & 5.51 & & 5 & & \\
\hline Log population & 2.60 & & 2.53 & & 2.21 & & 1.92 \\
\hline$k$-value & & 0.07 & & 0.32 & & 0.29 & \\
\hline
\end{tabular}

Numbers between parentheses represent the range of duration in days

The previously obtained results indicate that Nicola seems to be the least susceptible variety for infestation by Ph. operculella followed by Spunta and Simone. In contrast Atlas proved to be the most favorable variety. Many investigators studied the effect of variety on natural mortality and age structure data figures from which Ibrahim (2000) in Egypt revealed that larval stage exhibited the highest mortality rate at $25-30^{\circ} \mathrm{C}$ while this rate for egg stage was at $35^{\circ} \mathrm{C}$. Gurr \& Symington (1998) found that pickling the tuber surface immediately prior to inoculation with $\mathrm{Ph}$. operculella had lead to significant increase in neonate survival. They explained that tuber periderm may constitute a significant barrier to invasion. Golizadeh \& Razmjou (2010) mentioned that the lowest $r_{\mathrm{m}}$ value indicates that a certain variety of potato is insusceptible compared to other cultivars (in this research is Nicola). Again, Horgan et al. (2010) reported that resistance against $P h$. operculella in wild potato varieties was generally concentrated in tuber periderm or cortex-based. Unidentified cortexbased resistance factors in the varieties they studied reduced survival and increased larval and pupal developmental times. Von-Arx et al. (1987); Das et al. (1993); Debnath et al. (2000); Stein \& Vendramim (2000); Lopez \& Vendramim (2001) and Al-Omairy (2012) reached almost the same results.

\section{REFRENCES}

Abdel-Wahab, M. A.; F. A. Abdel-Galil; K. K. Mohamed and M. M. Soliman (1987). Some biological aspects of the potato tuber worm, Ph. operculella in Egypt. Assuit J. Agric. Sci., 18 (3): 363-365. 
Al-Omairy, Kh. O. (2012). Laboratory study of life tables of potato tuber moth, Phthorimaea operculella (Zeller) under constant temperature. The $11^{\text {th }}$ Conf. Agric. Dev. Res., Cairo, Egypt, 27-30 March, pp 162. (Abstract)

Al-Taweel, A. A.; N. S. Zeyab and M. K. El-Jaborry (2006). Reaction of some potato varieties to infestation by potato tuber moth, Phthorimaea operculella (Zeller). Arab J. Pl. Prot., 24: 53-55. (Arabic Language)

Anderwartha, H. G. and L. C. Birch (1984). The distribution and abundance of animals, 298-308. Univ. of Chicago and London.

Chandel; R. S.; K. Rajnish and N. P. Kashyab (2001). Bioecology of potato tube moth, Phthorimaea operculella Zeller in mid hills of Himachal Pradesh (2001). J. Ent. Res., 25 (3): 195-203.

Das, G. P.; E. D. Magallona; K. V. Raman and C. B. Adalla (1993). Growth and development of the potato tuber moth, Phthorimaea operculella (Zeller), on resistant and susceptible potato genotypes in storage. Philippine Entomol.; 9(1): $15-27$.

Douches, D.; W. Pet; D. Visser, J. Coombs; Kelly Zakra; Kimberly Flecher; G. Bothma; J. Brink; M. Koch and H. Quemada (2010). Field and storage evaluations of 'SpuntaG2' for resistance to potato tuber moth and agronomic performance. J. Amer. Soc. Hort. Sci., 135: 333-340.

Debnath, M. C.; J. N. Khaund; B. K. Borah and P. C. Sarmah (2000). The reaction of host plants on the biology of potato tuber moth, Phthorimaea operculella (Zeller). Res. on Crops. Gaurav Soc. Agric. Res. Information Centre, Hisar, India: 1(2): 196-200.

El-Saadany, G. B.; F. M. Mariy; F. M. Daoud and M. Y. Ibrahim (1998). Life table studies on potato tuber moth, Ph. operculella. Zagazig H. Agric. Res., 25 (4): 649-657.

Fenemore, P. G. (1980). Susceptibility of potato cultivars to potato tuber moth, Phthorimaea operculella Zell. (Lepidoptea:Gelechiidae). N. Z. J. Agric. Res., 23: 539-546.

Ghazala, E. M. A. (2005). Studies on potato tuber moth Phthorimaea operculella (Zeller). M. Sc. Thesis, Fac. Agric. Alex. Univ., 145 pp.

Golizadeh, A. and J. Razmjou (2010). Life table parameters of Phthorimaea operculella (Lepidoptera:Gelechiidae), feeding on tubers of six potato cultivars. J. Econ. Ent., 103 (3): 996-972.

Gurr, G. M. and C. A. Symington (1998). Resistance to the potato tuber moth, Phthorimaea operculella (Zeller) (Lepidoptea:Gelechiidae), in potato (Solanum tuberosum spp. tuberosum tuberosum L.) tubers. Australian J. Ent., 37(1): 49-51.

Horgan, F. G.; D. T. Quiring; A. Lagnaoui; A. R. Salas and Y. Pelletier (2010). Variations in resistance against Phthorimaea operculella in wild potato tubers. Entomol. Exp. Appli., 371 (3): 269-279.

Ibrahim, M. Y. (2000). Further ecological and biological studies on some potato pests in Egypt. Ph. D. Thesis, Fac. Agric., Ain Shams Univ., pp 236.

Khattab, M. M.; A. N. Fayad and R. H. G. Mikhael (1995). Relative susceptibility of potato varieties to infestation with insect pests. Annals Agric. Sci. Moshtohor, 33 (4): 1539-1552.

Lopes, M. T. and J. D. Vendramim (2001). Resistance of potato genotypes to the potato tuber moth Phthorimaea operculella (Zeller). [Portuguese]. Sci. Agric. Univ. Sao Paulo, Escola Superior Agric. "Luiz de Queiroz" Piracicaba, Brazil; 58 (2): 235-239. 
Randon, Silvia I. (2010). The potato tuber worm: A literature review of its biology, ecology and control. Am. J. Pot. Res., 87: 149-166.

Sileshi, G. and J. Teriessa (2001). Tuber damage by potato tuber moth, Phthorimaea operculella Zeller (Lepidoptera: Gelechiidae) in the field in Eastern Ethiopia. Internat. J. Pest Manag., 47 (2): 109-113.

Stein, C. P. and J. D. Vendramim (2000). Antibiosis of potato clones to Phthorimaea operculella (Zeller) (Lepidoptera: Gelechiidae). Anal. Soc. Entomol. Brazil; 29(4): 783-788.

Tiwari, D. B.; R. B. Thapa; S. M. Shrestha and S. L. Joshi (2006). Field survey and monitoring of potato tuber moth, Phthorimaea operculella (Zeller) (Lepidoptera: Gelechiidae). J. Inst. Agric. Anim. Sci., 27: 157-160.

Trivedi, T. P.; D. Rajagopal and P. L. Tandon (1994). Life table for establishment of potato tuber moth, Ph. operculella. J. Indian Potato Assoc., 12 (1-2): 97-105.

Von-Arx, R.; J. Goueder; M. Cheikh and T. A. Ben (1987). Integrated control of potato tuber moth Phthorimaea operculella (Zeller). Insect Sci. Appl. 8: 989994.

\section{ARABIC SUMMARY}

\section{معايير جداول الحياة كدليل لحساسية أصناف البطاطس للإصابة بفراشة درنات البطاطس Phthorimaea operculella (Zeller)}

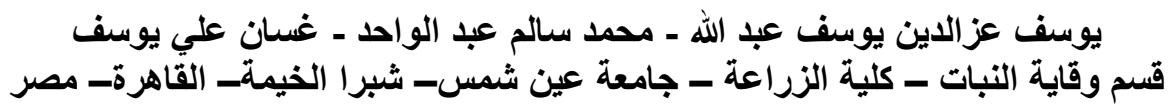

أجريت تجربة معملية لدر اسة جداول الحياة لفر اشة درنات البطاطس (Zeller)

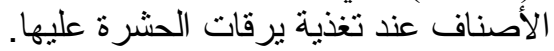

أظهرت النتائج أنه عند حساب معدلات البقاء الزباء و الخصوبة ومعايير جداول الحياة (معدل الزيادة الطبيعي،

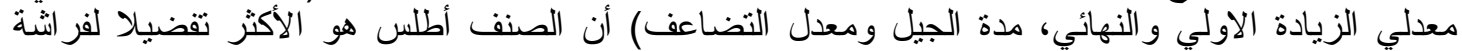

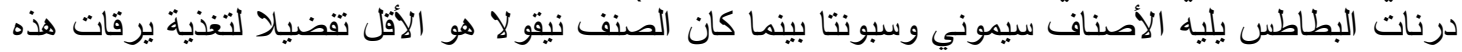

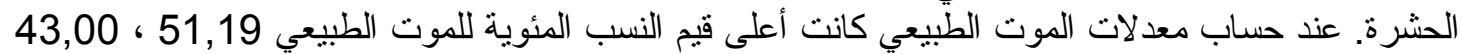

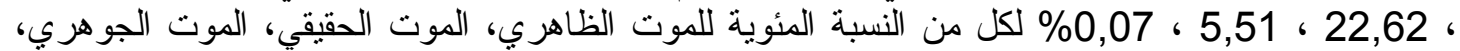

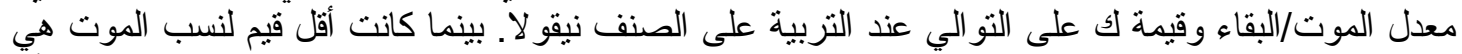

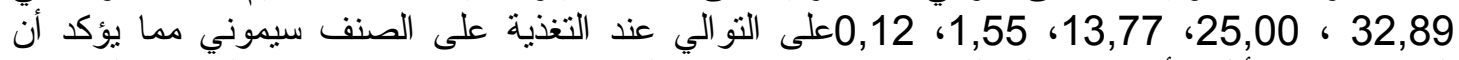

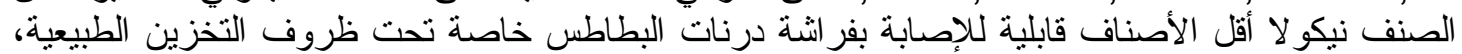

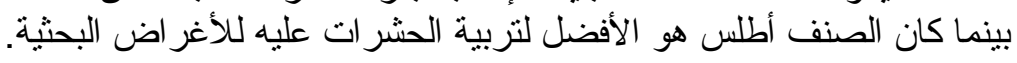

